\section{(6) OPEN ACCESS}

\title{
Discrepancies between patient-reported outcomes, and endoscopic and histological appearance in UC
}

\author{
Jean-Frédéric Colombel, ${ }_{1}^{1}$ Mary E Keir, ${ }^{2}$ Alexis Scherl, ${ }^{2}$ Rui Zhao, ${ }^{2}$ Gert de Hertogh, ${ }^{3}$ \\ William A Faubion, ${ }^{4}$ Timothy T Lu ${ }^{2}$
}

Additional material is published online only. To view please visit the journal online (http://dx.doi.org/10.1136/ gutjnl-2016-312307).

'Division of Gastroenterology, Icahn School of Medicine at Mount Sinai, New York, New York, USA

${ }^{2}$ Genentech Research and Early Development, South San Francisco, California, USA

${ }^{3}$ Department of Morphology and Molecular Pathology, University Hospital Gasthuisberg, Leuven, Belgium

${ }^{4}$ Division of Gastroenterology and Hepatology, Mayo Clinic, Rochester, Minnesota, USA

\section{Correspondence to}

Professor Jean-Frédéric

Colombel, Division of

Gastroenterology, Icahn School

of Medicine at Mount Sinai, 1

Gustave L. Levy Place, New York,

NY 10029, USA;

jean-frederic.colombel@mssm. edu

Received 23 May 2016 Revised 25 July 2016 Accepted 15 August 2016

Published Online First

2 September 2016

\section{ABSTRACT}

Objective Both endoscopy and histology may be included in the definition of mucosal healing in UC. This study aimed to establish the association between patient-reported outcomes, specifically symptom measures, and the presence of inflammation as measured by endoscopy and histology in UC.

Design Using patient data from an observational multicentre study of UC $(n=103)$, rectal bleeding (RB) and stool frequency (SF) symptom subscores of the Mayo Clinic Score (MCS) were compared with the endoscopic subscore (MCSe) and histology. Faecal calprotectin and biopsy cytokine expression were also evaluated.

Results When identifying UC patients with inactive disease, RB scores were superior to SF scores and the combination (sensitivity/specificity: MCSe=0/1, RB 77\%/ $81 \%$, SF 62\%/95\%, RB+SF 54\%/95\%; MCSe=0, RB $87 \% / 66 \%$, SF $76 \% / 83 \%$, RB+SF $68 \% / 86 \%$ ). Across different definitions of mucosal healing (MCSe $\leq 1 ; 0$; or 0 plus inactive histology), a larger subset of patients reported increased SF $(39 \%, 25 \%$ and $27 \%$, respectively) compared with RB (24\%, $13 \%$ and $10 \%)$. Faecal calprotectin and inflammatory cytokine expression were higher in patients with active disease compared with patients with mucosal healing, but there were no differences between patients using increasingly stringent definitions of mucosal healing.

Conclusions Endoscopically inactive disease is associated with absence of RB but not with complete normalisation of SF. Achieving histological remission did not improve symptomatic relief. In addition, in these patients, higher inflammatory biomarker levels were not observed. These data suggest that non-inflammatory changes, such as bowel damage, may contribute to SF in UC.

\section{INTRODUCTION}

UC is a chronic inflammatory disease characterised by mucosal ulceration of the colon and symptoms that include diarrhoea and bleeding from the rectum. The clinical activity of UC can vary from severe symptoms to a milder disease course, with patients experiencing periodic disease flares that spontaneously resolve or can be brought under control with therapeutic intervention. ${ }^{1}$ Rectal bleeding $(\mathrm{RB})$ and diarrhoea are commonly used to monitor disease activity in patients with UC in both clinical practice and clinical trials. Blood in stools is reported by $>90 \%$ of UC patients with active disease and is associated with ulceration of mucosa

\section{Significance of this study}

What is already known on this subject?

- Previous studies have shown discrepancies between endoscopic healing and presence of symptoms in patients with UC.

- Persistence of symptoms in patients with endoscopic healing may be related to ongoing histological activity.

- Regulatory definitions of mucosal healing are moving towards inclusion of endoscopy and histology.

- Associations between patient-reported outcomes and endoscopy and histology have not been established.

What are the new findings?

- Across increasingly stringent definitions of mucosal healing, a larger subset of patients reported abnormal stool frequency compared with rectal bleeding.

- Histological activity does not explain the persistence of symptoms in UC patients with endoscopic remission.

- UC biomarker levels did not differ between patients categorised under different definitions of mucosal healing, with or without histological healing.

How might it impact on clinical practice in the foreseeable future?

- Achieving complete remission of symptoms even in the presence of endoscopic and histological healing may be difficult with one therapy.

- Histological evaluation may not provide additional benefit for those patients who show inactive disease on endoscopic evaluation.

and loss of epithelial integrity. An increase in diarrhoea or stool frequency (SF) is the most common presenting symptom of UC. ${ }^{2}$ How symptoms of UC correlate with evidence of inflammation as measured by visual and microscopic examination of the gut lumen is incompletely understood. A recent report shows that resolution of clinical symptoms often, but not always, follows endoscopic healing. ${ }^{3}$ One hypothesis is that the persistence of histological inflammation could still drive symptomatic disease in these patients. 
Histological evaluation of inflammation in patients with endoscopic healing has shown that some patients have residual active inflammatory infiltrate despite macroscopically normal mucosa. ${ }^{4}$ Endoscopic resolution of disease following biological therapy has been shown to predict favourable outcomes, ${ }^{5}$ but the importance of histological healing as defined by the absence of active inflammation in patients with endoscopic healing has not yet been established. One barrier to using histology in patients with endoscopic healing has been the lack of a validated scoring system. Histological assessment is routinely performed in the clinic, and recent studies have sought to improve upon previously published descriptive measures. ${ }^{4}$ The robustness of these new scoring systems ${ }^{78}$ will help define the importance of active inflammation in patient outcomes.

Whether active histological inflammation in patients with endoscopic healing drives the persistence of symptoms in a subset of patients has not been evaluated. We sought to examine the relationship of symptoms to objective measures of inflammation in patients that continue to have symptoms, employing a gradually stricter definition of mucosal healing: endoscopy alone or a combination of endoscopy and histology. Faecal calprotectin (FCP), C reactive protein (CRP) and inflammatory cytokine expression, known biomarkers of inflammation, ${ }^{9} 10$ were also evaluated in mucosal biopsies from these patients. Improved understanding of the relationship of symptoms to mucosal healing as assessed by both endoscopy and histology may help guide treatment initiation and evaluation of therapeutic efficacy in UC.

\section{METHODS}

\section{Patients and study design}

The EMerging BiomARKers in Inflammatory Bowel Disease (EMBARK) cohort $^{11}$ consisted of patients with UC $(n=103)$ enrolled into an observational study with no therapeutic intervention. Patients were required to have a prior diagnosis of UC and complete, interpretable endoscopic data as determined by a central reader. All patients underwent a full ileocolonoscopy.

\section{End points}

Clinical disease activity was assessed using the Mayo Clinic Score (MCS). RB, SF and Mayo endoscopic subscores (MCSe) are separate components of the MCS. MCSe subscores were assessed by a central reader using still images and video as described. ${ }^{11}$ Histology scoring was performed on biopsies taken from the most inflamed area of the colon, $10-40 \mathrm{~cm}$ from the anal verge. Biopsies of ulcerated mucosa or suture sites were avoided. A single pathologist $(\mathrm{GdH})$ scored the biopsies using the Geboes score; ${ }^{6}$ scores were then converted to the Robarts histopathology index (RHI) score ${ }^{8}$ and the Nancy index score ${ }^{7}$ (see online supplementary table S1).

\section{Biomarker analysis}

FCP and CRP were measured as previously described. ${ }^{11}$ At the time of endoscopy, the endoscopist collected intestinal biopsies from patients with UC. In patients with active disease, biopsies from inflamed areas were used for analysis; in patients without active disease $(\mathrm{MCSe}=0)$, uninflamed biopsies were used. The biopsy samples were homogenised with $3 \mathrm{~mm}$ steel beads using a TissueLyzer (Qiagen, Germantown, Maryland, USA) and RNA was isolated using the RNeasy kit (Qiagen). RNA integrity was assessed with the Agilent 2100 Bioanalyzer using the Agilent RNA 6000 Pico Kit (Agilent Technologies, Santa Clara, California, USA). Samples with high RNA quality (RNA integrity number $>5)$ were included in the analysis $(n=66)$.
Reactions were performed using the BioMark HD System (Fluidigm, South San Francisco, California, USA) using human tumour necrosis factor (TNF)- $\alpha$, interleukin (IL)-6, IL-8, IL-1 $\beta$ and glyceraldehyde 3-phosphate dehydrogenase (GAPDH) primer sets (Applied Biosystems, Foster City, California, USA). IL-6, IL-8, IL-1 $\beta$ and TNF- $\alpha$ expression was normalised to GAPDH and a reference human RNA sample using the $\Delta \mathrm{Ct}$ method.

\section{Statistical methods}

A retrospective, post hoc analysis of data collected in the EMBARK study was performed. None of the outcomes evaluated here were prespecified. Comparisons between groups were performed assuming classification by the MCSe as the reference standard. Three definitions of mucosal healing were studied: two definitions used endoscopy alone $(\mathrm{MCSe}=0$, completely normal mucosa; $\mathrm{MCSe}=0 / 1$, inactive or mild disease with no ulcers), while the third used both endoscopy and histology (MCSe $=0$, with inactive histology defined as RHI $\leq 6$ and Nancy $\leq 1)$. The RB and SF subscores were considered as the test scores in all analyses. The positive predictive value (PPV), negative predictive value (NPV), sensitivity and specificity of RB and SF subscores were calculated for each definition separately for RB subscore $=0, \mathrm{SF}$ subscore $=0$, and $\mathrm{RB}+\mathrm{SF}$ subscore $=0$.

The PPV was defined as the proportion of patients with mucosal healing when the test/subscore $=0$. The NPV was defined as the proportion of patients without mucosal healing when the test/subscore $>0$. Sensitivity of a symptom score was defined as the proportion of patients with the test $/$ subscore $=0$ when mucosal healing was present according to endoscopy. Specificity of a symptom score was defined as the proportion of patients with the test/subscore $>0$ when mucosal healing was not present according to endoscopy.

\section{RESULTS}

\section{Patient demographics and baseline characteristics}

The patient baseline characteristics have been previously described. ${ }^{11}$ Disease severity, as measured by the total MCS, was mild in the EMBARK patient population (median $=4$, mean $=4.5 \pm 3.8)$ with $\sim 60 \%(61 / 103)$ of patients having inactive disease as measured by endoscopy.

\section{Relationship of RB and SF, and endoscopic healing}

Figure 1 summarises the distribution of MCSe subscores across increasing RB and SF subscores. In EMBARK, 60\% (33/55) and $73 \%(29 / 40)$ of patients that reported RB or SF scores of 0 had an MCSe score of 0 , respectively. In patients reporting an RB or SF score of 1, 19\% (4/21) and 32\% (6/19) had an MCSe score of 0 . With increasing symptom scores, the proportion of patients without evidence of endoscopic activity $(\mathrm{MCSe}=0)$ decreased, but a proportion of patients without endoscopic activity still had more severe symptoms $(\mathrm{RB}=2: 5 \%, 1 / 22$; $\mathrm{SF}=2: 18 \%, 3 / 17)$.

When endoscopic healing was defined as MCSe 0 or 1 , the sensitivities and specificities of $\mathrm{RB}, \mathrm{SF}$ and $\mathrm{RB}+\mathrm{SF}$ for detecting mucosal healing were $77 \%$ and $81 \%, 62 \%$ and $95 \%$, and $54 \%$ and $95 \%$, respectively (table 1 ). When a more stringent definition of endoscopic healing was used $(\mathrm{MCSe}=0)$, overall sensitivities of the symptom subscores increased and specificities decreased (table 2).

When endoscopic healing was defined as MCSe 0 or 1 , the PPVs for RB, SF and RB+SF were $86 \%, 95 \%$ and $94 \%$, respectively. The NPVs for RB, SF and RB+SF were 71\%, 64\% and $59 \%$, respectively (table 1). When endoscopic healing was 

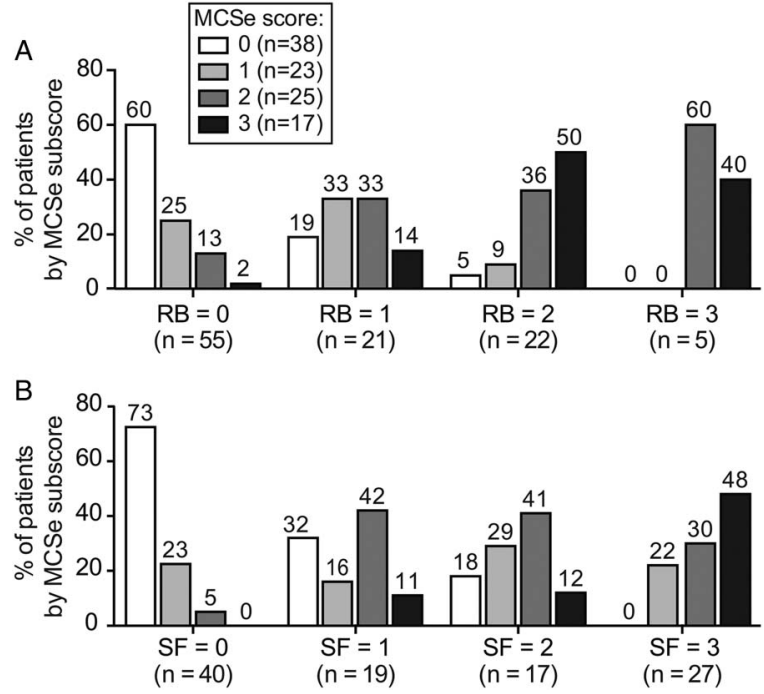

Figure 1 Proportion of patients with Mayo endoscopic subscores (MCSe) $=0-3$ by increasing $(A)$ rectal bleeding (RB) and (B) stool frequency (SF) subscores.

defined as $\mathrm{MCSe}=0$, overall PPVs for each symptom score decreased and NPVs increased (table 2).

\section{Evaluation of symptoms in patients with mucosal healing using endoscopic and histological measures}

In addition to using endoscopic measures of mucosal healing, symptoms were evaluated using a more stringent definition that incorporated both endoscopic and histological measures of disease activity. When we assessed histology with the Nancy ${ }^{7}$ and Robarts ${ }^{8}$ histopathology indices in patients with an endoscopy score of 0 , each score identified the same patients as inactive.

Across the different definitions of mucosal healing $(\mathrm{MCSe} \leq 1$, $\mathrm{MCSe}=0$ or $\mathrm{MCSe}=0$ /inactive histology), the percentage of patients with abnormal SF (SF>0) was higher than the corresponding percentage of patients with $\mathrm{RB}(\mathrm{RB}>0$; figure 2$)$. In patients with MCSe of 0 or $1,24 \%$ of patients reported an RB score $\geq 1$, while $6 \%$ had an $R B$ score $\geq 2$. In the same patient group, $39 \%$ of patients had an SF score $\geq 1$ and $25 \%$ reported an $S F \geq 2$. A similar trend was observed in patients with $\mathrm{MCSe}=0 \quad(13 \%$ with $\mathrm{RB} \geq 1,3 \%$ with $\mathrm{RB} \geq 2 ; 25 \%$ with $\mathrm{SF} \geq 1$ and $9 \%$ with $\mathrm{SF} \geq 2$ ). For those patients with $\mathrm{MCSe}=0$ /inactive histology, the proportion reporting abnormal $\mathrm{SF}$ was higher than the proportion reporting $\mathrm{RB}(\mathrm{RB} \geq 1,10 \%$; $\mathrm{SF} \geq 1,27 \%$; $\mathrm{RB} \geq 2,3 \% ; \mathrm{SF} \geq 2,10 \%)$. Notably, the majority of patients with
$\mathrm{MCSe}=0$ had inactive histology, and the addition of histological inactivity to endoscopy did not change the rate of patients reporting symptoms.

No differences in corticosteroid, immunosuppressant use, disease duration or extent of disease were found in patients with inactive disease irrespective of symptoms (data not shown).

\section{Biomarker analysis in patients with mucosal healing using endoscopic and histological measures}

Biomarker correlates of inflammation, including FCP and CRP, have also been used to follow disease activity in clinical studies of UC. $^{9}{ }^{10}$ Therefore, we evaluated levels of FCP (figure 3A) and serum CRP (see online supplementary figure S1) in patients with UC based on different definitions of mucosal healing (MCSe $\leq 1$, $\mathrm{MCSe}=0$, or $\mathrm{MCSe}=0$ /inactive histology). Patients with endoscopy scores of 2 or 3 had significantly higher levels of FCP in comparison to patients with all definitions of mucosal healing $(\mathrm{p}<0.01$; mean $\pm \mathrm{SD}$ concentrations: $\mathrm{MCSe} \geq 2,869 \pm 782 \mu \mathrm{g} / \mathrm{g}$; MCSe $\leq 1,319 \pm 440 \mu \mathrm{g} / \mathrm{g} ; \mathrm{MCSe}=0,263 \pm 418 \mu \mathrm{g} / \mathrm{g} ; \mathrm{MCSe}=0$ +inactive histology, $247 \pm 426 \mu \mathrm{g} / \mathrm{g}$ ). CRP was also higher in patients with $\mathrm{MCSe} \geq 2$ in comparison to patients with mucosal healing $(\mathrm{p}<0.01$; mean \pm SD concentrations: $\mathrm{MCSe} \geq 2$, $1.95 \pm 0.3 \mathrm{mg} / \mathrm{dL} ; \quad M C S e \leq 1, \quad 0.8 \pm 0.1 \mathrm{mg} / \mathrm{dL} ; \quad \mathrm{MCSe}=0$, $0.64 \pm 0.1 \mathrm{mg} / \mathrm{dL} ; \mathrm{MCSe}=0 /$ inactive histology, $0.65 \pm 0.1 \mathrm{mg} / \mathrm{dL}$ ). Similar to symptom scores, the addition of histology to patients with an $\mathrm{MCSe}=0$ did not result in significant differences in CRP or FCP.

Low FCP levels are associated with the absence of histological inflammation ${ }^{12}$ and the likelihood of achieving clinical remission. ${ }^{10}$ We therefore evaluated symptom scores in patients using a definition of mucosal healing that incorporated low $(\leq 150 \mu \mathrm{g} / \mathrm{g})$ levels of FCP in addition to endoscopic and histological measures (figure 3B, C). Only a small number of patients with no evidence of endoscopic disease activity $(\mathrm{MCSe}=0)$ reported $\mathrm{RB}$ $(\mathrm{RB} \geq 1,7 \%)$, while a significant fraction reported abnormal SF $(\mathrm{SF} \geq 1,29 \% ; \mathrm{SF} \geq 2,21 \%)$. The percentage of patients with symptoms was similar when patients with active histology were removed from the analysis.

A subset of patients with $\mathrm{MCSe}=0$ and no active histology had biopsies available for analysis of inflammatory cytokines. Patients with high endoscopy scores $(\mathrm{MCS} \geq 2)$ had higher levels of IL-6, IL-8, IL-1 $\beta$ and TNF- $\alpha$ in comparison to patients with $\mathrm{MCSe}=0$ (data not shown). Although the numbers were small, there were no differences in cytokine expression between patients with an MCSe of 0 and $\mathrm{SF} \geq 1$ in comparison to patients with an MCSe of 0 who did not report increased SF (see online supplementary figure S2).

Table 1 Comparison of rectal bleeding (RB) and stool frequency (SF) to endoscopic healing as defined by Mayo endoscopic subscores (MCSe)=0/1

\begin{tabular}{|c|c|c|c|c|c|c|}
\hline \multirow[b]{2}{*}{ Symptom score } & \multicolumn{2}{|l|}{ MCSe } & \multirow[b]{2}{*}{ PPV } & \multirow[b]{2}{*}{ NPV } & \multirow[b]{2}{*}{ Sensitivity } & \multirow[b]{2}{*}{ Specificity } \\
\hline & $=0 / 1$ & $\geq 2$ & & & & \\
\hline $\mathrm{RB}=0$ & 47 & 8 & $85.5 \%(73.3-96.5)$ & $70.8 \%(55.9-83.0)$ & $77.0 \%(64.5-86.8)$ & $81.0 \%(65.9-91.4)$ \\
\hline $\mathrm{RB}>0$ & 14 & 34 & & & & \\
\hline $\mathrm{SF}=0$ & 38 & 2 & $95.0 \%(83.1-99.4)$ & $63.5 \%(50.4-75.3)$ & $62.3 \%(49.0-74.4)$ & $95.2 \%(83.8-99.4)$ \\
\hline $\mathrm{SF}>0$ & 23 & 40 & & & & \\
\hline $\mathrm{RB}+\mathrm{SF}=0$ & 33 & 2 & $94.3 \%(80.8-99.3)$ & $58.8 \%(46.2-70.6)$ & $54.1 \%(40.8-66.9)$ & $95.2 \%(83.8-99.4)$ \\
\hline $\mathrm{RB}+\mathrm{SF}>0$ & 28 & 40 & & & & \\
\hline
\end{tabular}


Table 2 Comparison of rectal bleeding (RB) and stool frequency (SF) to endoscopic healing as defined by Mayo Clinic endoscopic subscores (MCSe) $=0$

\begin{tabular}{|c|c|c|c|c|c|c|}
\hline \multirow[b]{2}{*}{ Symptom score } & \multicolumn{2}{|c|}{ MCSe } & \multirow[b]{2}{*}{ PPV } & \multirow[b]{2}{*}{ NPV } & \multirow[b]{2}{*}{ Sensitivity } & \multirow[b]{2}{*}{ Specificity } \\
\hline & $=0$ & $\geq 1$ & & & & \\
\hline $\mathrm{RB}=0$ & 33 & 22 & $60.0 \%(45.9-73.0)$ & $89.6 \%(77.3-96.5)$ & $86.8 \%$ (71.9-95.6) & $66.2 \%(53.4-77.4)$ \\
\hline $\mathrm{RB}>0$ & 5 & 43 & & & & \\
\hline $\mathrm{SF}=0$ & 29 & 11 & $72.5 \%(56.1-85.4)$ & $85.7 \%(74.6-93.3)$ & $76.3 \%(59.8-88.6)$ & $83.1 \%(71.7-91.2)$ \\
\hline $\mathrm{SF}>0$ & 9 & 54 & & & & \\
\hline $\mathrm{RB}+\mathrm{SF}=0$ & 26 & 9 & $74.3 \%(56.7-87.5)$ & $82.4 \%(71.2-90.5)$ & $68.4 \%(51.3-82.5)$ & $86.2 \%(75.3-93.5)$ \\
\hline $\mathrm{RB}+\mathrm{SF}>0$ & 12 & 56 & & & & \\
\hline
\end{tabular}

No. of patients are provided for each score; PPV, NPV, sensitivity and specificity values are $\%(95 \% \mathrm{Cl})$.

NPV, negative predictive value; PPV, positive predictive value.
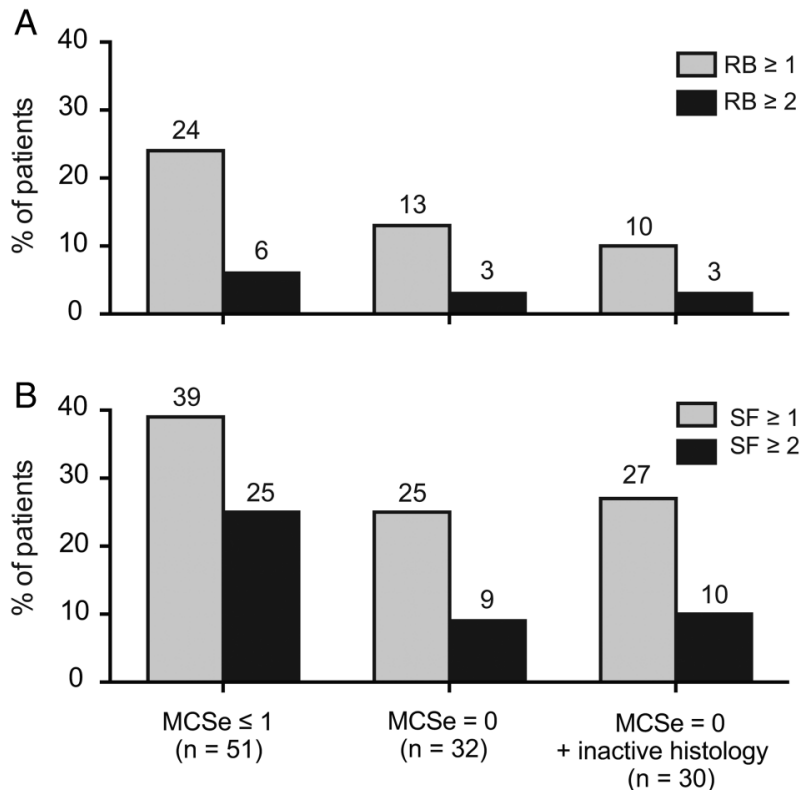

Figure 2 Proportion of patients with $(A)$ rectal bleeding (RB) and (B) stool frequency (SF) symptoms, grouped by increasingly stringent definitions of mucosal healing. MCSe, Mayo endoscopic subscores.

\section{DISCUSSION}

Recently, the Selecting Therapeutic Targets in Inflammatory Bowel Disease programme has recommended a 'treat-to-target' approach for UC that includes using a combination of patientreported outcomes (PROs) and objective measures such as endoscopy as treatment and clinical trial end points. ${ }^{13}$ Previous studies, however, have found discrepancies between endoscopy findings and PROs. ${ }^{3}{ }^{10}$ Using data from EMBARK, an observational study of patients with UC, we evaluated the PROs of SF and $\mathrm{RB}$ against increasingly strict definitions of mucosal healing that included endoscopic or endoscopic and histological components. Consistent with others' results, ${ }^{3} 10$ we found that symptom scores generally correlated well with endoscopic scores, but some patients still exhibited persistent symptoms while having endoscopic scores of 0 or 1 . Additionally, as the stringency of the mucosal healing definition increased $(\mathrm{MCS} e \leq 1$ vs $\mathrm{MCSe}=0$ ), fewer patients exhibited symptoms; however, the proportion of patients exhibiting abnormal SF was consistently greater than the proportion with RB.

Findings from this study and previous studies ${ }^{3} 10$ suggested that the presence of histological inflammation in the setting of endoscopic healing as potentially one explanation for the persistence of symptoms. We therefore extended the mucosal healing definition to include patients with inactive disease according to histology using the newly validated Robarts ${ }^{8}$ and $\mathrm{Nancy}^{7}$ histology indices. Adding histological assessment, however, did not alter the proportion of patients reporting symptoms. Furthermore, FCP, CRP and inflammatory gene expression levels did not differ between the different mucosal healing definitions with or without histological healing. It should be noted that although we observed agreement between the indices in identifying patients with inactive disease and an endoscopy score of 0 , discordance in histological disease activity was observed due to the presence of neutrophils.

While these symptoms have been attributed to IBS, the underlying cause of persistently increased SF in patients who show both endoscopic and histological healing may lie elsewhere. While inflammation and ulceration in UC is limited to the mucosa and superficial submucosa, chronic inflammation in UC leads to marked distortion of the anatomy of the entire bowel wall, including mucosal atrophy and muscular changes that include fibromuscular dysplasia of the muscularis mucosae and atrophy of the muscularis propria. These changes to the muscular organisation of the bowel wall reflect structural, and likely functional, bowel damage that may manifest as decreased motility, narrowing of the rectum and the colon, and changes in absorptive function. ${ }^{14}$ Although fibrosis, the excessive deposition of extracellular matrix proteins beyond the mucosal layer, is more common in Crohn's disease, fibrosis in patients with UC may lead to stiffness of the intestinal wall, problems with colonic motility and anorectal dysfunction, often without any visible inflammation, ${ }^{14-16}$ and may explain increased SF symptoms. Inflammation may also directly damage the enteric nervous system and affect GI motility ${ }^{17} 18$ or have indirect effects on the microbiota. Altered microbiota may affect serotonin production by enterochromaffin cells and induce modifications in GI motility. ${ }^{19}$

The current study not only employed the most commonly used definition of mucosal healing $(\mathrm{MCSe}=0 / 1)$, but also included more rigorous definitions of complete endoscopic remission $(\mathrm{MCSe}=0)$ and endoscopic remission plus inactive histology. For both endoscopy and histology analyses, we used central readers, which eliminated the biases in scoring that may have occurred had they been read locally. Histology was also scored using the most recently validated indices, which agreed with each other in terms of patient disease status. However, this study had some limitations. Our cohort size was limited $(n=103)$ and included patients with inactive and active disease. 

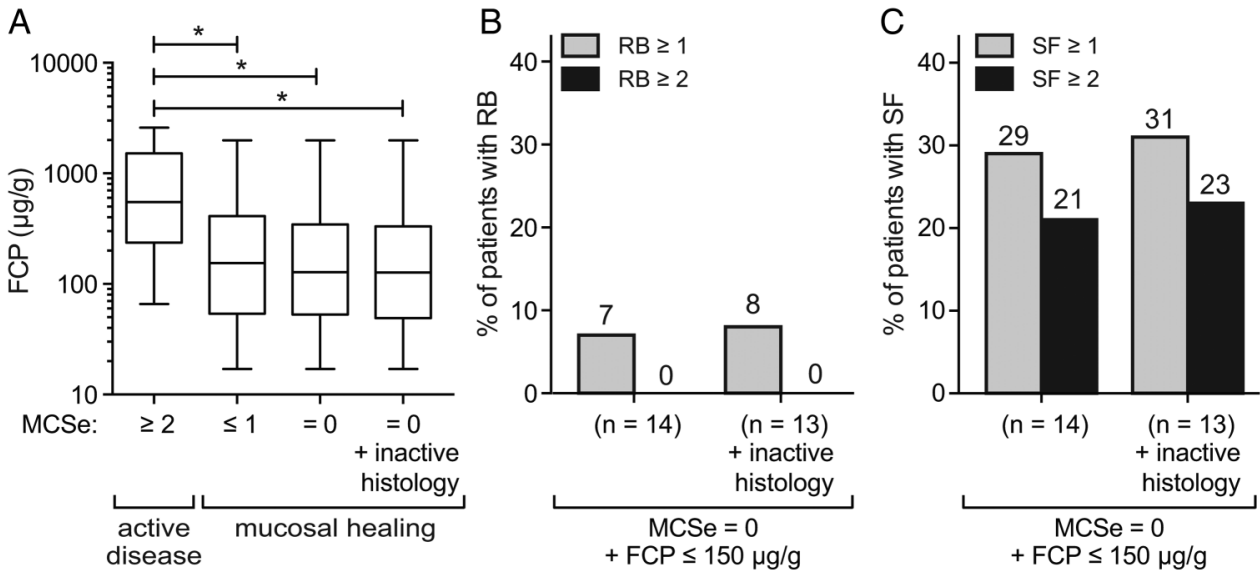

Figure 3 Faecal calprotectin (FCP) levels and symptom scores. (A) Box plots (median IQR) showing FCP levels in patients with active disease (Mayo endoscopic subscores (MCSe) $\geq 2$ ) or different definitions of mucosal healing (MCSe $\leq 1, \mathrm{MCSe}=0, \mathrm{MCS} e=0 /$ inactive histology). ${ }^{*} \mathrm{p}<0.01$ by the Kruskal-Wallis test. Proportion of patients with (B) rectal bleeding (RB) and (C) stool frequency (SF) symptoms who have low FCP ( $\leq 150 \mu \mathrm{g} / \mathrm{g})$ and mucosal healing (MCSe=0 or MCSe=0/inactive histology).

Because this population had relatively mild disease (median MCS =4) and the majority of patients $(59 \%)$ had $\mathrm{MCSe} \leq 1$, these results may not be generalisable to a clinical trial population. This retrospective analysis also had limited statistical power, as evidenced by the width of the CIs. However, similar trends in symptom scores and discrepancies between SF and endoscopic findings recently reported in a clinical study ${ }^{3}$ support that these findings are consistent across both patients with recent disease activity and those patients with milder disease.

In conclusion, our results have important implications for both clinical practice and the design of clinical trials. Our work highlights the possibility that it may be very difficult for a therapy to achieve complete remission of symptoms even in the presence of endoscopic and histological healing. Furthermore, we do not observe additional value by using histological evaluation in patients with no evidence of disease on endoscopic evaluation, but longitudinal outcomes studies will be required to completely evaluate the value of histology. Given that the persistence of symptoms greatly impacts patient quality of life, it is vital to understand the underlying mechanisms that drive them and eventually work towards treatment regimens that can address both mucosal healing and symptom relief.

Acknowledgements Editing and writing assistance was provided by Deborah Solymar (Genentech) and was funded by Genentech.

Contributors Genentech was involved in the study design, data interpretation and the decision to submit for publication in conjunction with the authors. J-FC conceived the current analysis and interpreted the data. MEK and TTL conceived the current analysis, and analysed and interpreted the data. AS analysed and interpreted the data. RZ and GdH analysed the data. WAF contributed to the design of the EMBARK study and acquired the data. All authors contributed to the drafting and revision of the manuscript and approved the final version.

Funding This study was funded by Genentech.

Competing interests J-FC has received research funding Abbvie, Genentech, Janssen and Janssen, and Takeda and has served as a consultant for Abbvie, Amgen, Boehringer Ingelheim, Celgene Corporation, Celltrion, Enterome, Ferring, Genentech, Janssen Pharmaceuticals, Medimmune, Merck \& Co., Pfizer, Protagonist, Second Genome, Seres, Takeda and Theradiag. He has also been a speaker for Abbvie, Ferring, Takeda and Shire. He has received stock options from Intestinal Biotech Development and Genfit. MEK, AS, RZ and TTL are employees of Genentech, a member of the Roche group and own Roche stock. GdH has received payment to his institution from Genentech for the central pathology review in this manuscript and his institution has received payment for his central pathology review services from Shire Novartis, Centocor and Galapagos. WAF serves as an advisory board member for
Abbvie, Janssen Pharmaceuticals and Boehringer Ingelheim Pharma and serves as a consultant for Celgene, Connecticut Children's Medical Center, Genentech, Janssen Pharmaceuticals, Shire and Velocity Pharmaceutical Development.

Ethics approval Atlanta Gastroenterology Assoc; Borland-Groover Clinic; London Health Sciences Center; Mayo Clinic; Mount Sinai School of Medicine; UNC at Chapel Hill School Med; Univ of Chicago Hospitals; University of Florida College of Med; University of Kentucky; University of Michigan; Vancouver Liver and Intestinal Research Center; Wisconsin Center Advanced Research; Yale Department of Digestive Disease. The objective of this study was to assess various biomarkers for correlation with objective measures of disease activity. All assessments were done at baseline. Institutional review boards at each study site approved the protocols, and all patients provided written informed consent.

Provenance and peer review Not commissioned; externally peer reviewed.

Open Access This is an Open Access article distributed in accordance with the Creative Commons Attribution Non Commercial (CC BY-NC 4.0) license, which permits others to distribute, remix, adapt, build upon this work non-commercially, and license their derivative works on different terms, provided the original work is properly cited and the use is non-commercial. See: http://creativecommons.org/ licenses/by-nc/4.0/

\section{REFERENCES}

1 Danese S, Fiocchi C. Ulcerative colitis. N Engl J Med 2011:365:1713-25.

2 Ordás I, Eckmann L, Talamini M, et al. Ulcerative colitis. Lancet 2012;380:1606-19.

3 Jharap B, Sandborn WJ, Reinisch W, et al. Randomised clinical study: discrepancies between patient-reported outcomes and endoscopic appearance in moderate to severe ulcerative colitis. Aliment Pharmacol Ther 2015;42:1082-92.

4 Riley SA, Mani V, Goodman MJ, et al. Microscopic activity in ulcerative colitis: what does it mean? Gut 1991:32:174-8.

5 Colombel JF, Rutgeerts P, Reinisch W, et al. Early mucosal healing with infliximab is associated with improved long-term clinical outcomes in ulcerative colitis. Gastroenterology 2011;141:1194-201.

6 Geboes K, Riddell R, Ost A, et al. A reproducible grading scale for histological assessment of inflammation in ulcerative colitis. Gut 2000;47:404-9.

7 Marchal-Bressenot A, Salleron J, Boulagnon-Rombi C, et al. Development and validation of the Nancy histological index for UC. Gut. Published Online First: 13 Oct 2015. doi:10.1136/gutjnl-2015-310187

8 Mosli MH, Feagan BG, Zou G, et al. Development and validation of a histological index for UC. Gut. Published Online First: 16 Oct 2015. doi:10.1136/gutjnl-2015310393

9 Boland BS, Boyle DL, Sandborn WJ, et al. Validation of gene expression biomarker analysis for biopsy-based clinical trials in Crohn's disease. Inflamm Bowel Dis 2015;21:323-30

10 Sandborn WJ, Panés J, Zhang $\mathrm{H}$, et al. Correlation between concentrations of fecal calprotectin and outcomes of patients with ulcerative colitis in a phase 2 trial. Gastroenterology 2016;150:96-102.

11 Faubion WAJr, Fletcher JG, O'Byrne $\mathrm{S}$, et al. EMerging BiomARKers in Inflammatory Bowel Disease (EMBARK) study identifies fecal calprotectin, serum MMP9, and serum IL-22 as a novel combination of biomarkers for Crohn's disease activity: role of cross-sectional imaging. Am I Gastroenterol 2013;108:1891-900. 
12 Zittan E, Kelly $\mathrm{OB}$, Kirsch $\mathrm{R}$, et al. Low fecal calprotectin correlates with histological remission and mucosal healing in ulcerative colitis and colonic Crohn's disease. Inflamm Bowel Dis 2016;22:623-30.

13 Peyrin-Biroulet L, Sandborn W, Sands BE, et al. Selecting therapeutic targets in inflammatory bowel disease (STRIDE): determining therapeutic goals for treat-to-target. Am J Gastroenterol 2015;110:1324-38.

14 Torres J, Billioud V, Sachar DB, et al. Ulcerative colitis as a progressive disease: the forgotten evidence. Inflamm Bowel Dis 2012;18:1356-63.

15 Latella G, Di Gregorio J, Flati V, et al. Mechanisms of initiation and progression of intestinal fibrosis in IBD. Scand J Gastroenterol 2015;50:53-65.
16 Latella G, Rieder F. Time to look underneath the surface: ulcerative colitis-associated fibrosis. J Crohns Colitis 2015;9:941-2.

17 Geboes K, Collins S. Structural abnormalities of the nervous system in Crohn's disease and ulcerative colitis. Neurogastroenterol Motil 1998; 10: 189-202.

18 Bernardini N, Segnani C, Ippolito C, et al. Immunohistochemical analysis of myenteric ganglia and interstitial cells of Cajal in ulcerative colitis. J Cell Mol Med 2012;16:318-27.

19 Yano JM, Yu K, Donaldson GP, et al. Indigenous bacteria from the gut microbiota regulate host serotonin biosynthesis. Cell 2015;161:264-76. 